\title{
A!
}

This is an electronic reprint of the original article.

This reprint may differ from the original in pagination and typographic detail.

Dube, M.; Majaniemi, S.; Rost, M.; Alava, MJ; Elder, KR; Ala-Nissilä, Tapio

\section{Interface pinning in spontaneous imbibition}

Published in:

Physical Review E

DOI:

10.1103/PhysRevE.64.051605

Published: 01/11/2001

Document Version

Publisher's PDF, also known as Version of record

Please cite the original version:

Dube, M., Majaniemi, S., Rost, M., Alava, MJ., Elder, KR., \& Ala-Nissilä, T. (2001). Interface pinning in spontaneous imbibition. Physical Review E, 64(5), 1-6. [051605]. https://doi.org/10.1103/PhysRevE.64.051605

This material is protected by copyright and other intellectual property rights, and duplication or sale of all or part of any of the repository collections is not permitted, except that material may be duplicated by you for your research use or educational purposes in electronic or print form. You must obtain permission for any other use. Electronic or print copies may not be offered, whether for sale or otherwise to anyone who is not an authorised user. 


\title{
Interface pinning in spontaneous imbibition
}

\author{
M. Dubé, ${ }^{1}$ S. Majaniemi, ${ }^{2,3}$ M. Rost, ${ }^{4}$ M. J. Alava,${ }^{3}$ K. R. Elder, ${ }^{5}$ and T. Ala-Nissila ${ }^{2,3}$ \\ ${ }^{1}$ Centre for the Physics of Materials, McGill University, Montréal, Québec, Canada H3A 2T8 \\ ${ }^{2}$ Helsinki Institute of Physics, P.O. Box 64, University of Helsinki, FIN-00014, Helsinki, Finland \\ ${ }^{3}$ Laboratory of Physics, P.O. Box 1100, Helsinki University of Technology, FIN-02015 HUT, Espoo, Finland \\ ${ }^{4}$ Institut für Theoretische Physik, Universität zu Köln, 50937 Köln, Germany \\ ${ }^{5}$ Department of Physics, Oakland University, Rochester, Michigan 48309-4487
}

(Received 1 May 2001; published 23 October 2001)

\begin{abstract}
Evaporation and gravity induced pinning in spontaneous imbibition are examined within a phase field formalism. Evaporation is introduced via a nonconserving term and gravity through a convective term that constrains the influx of liquid. Their effects are described by dimensionless coupling constants $\epsilon$ and $g$, respectively. From liquid conservation, the early time behavior of the average interface position follows $H(t) \sim t^{1 / 2}$ until a crossover time $t^{*}(g, \epsilon)$. After that the pinning height $H_{p}(g, \epsilon)$ is approached exponentially in time, in accordance with mean field theory. The statistical roughness of the interface is described by an exponent $\chi \simeq 1.25$ at all stages of the rise, but the dynamic length scale controlling roughness crosses over from $\xi_{\times} \sim H^{1 / 2}$ to a time independent pinning length scale $\xi_{p}(\epsilon, g)$.

DOI: 10.1103/PhysRevE.64.051605

PACS number(s): 68.35.Ct, 68.35.Fx, 47.55.Mh, 05.70.Ln
\end{abstract}

\section{INTRODUCTION}

The propagation and possible pinning of interfaces moving in quenched random media provide several theoretical and experimental challenges with many questions still unanswered. Based on the presence or absence of a conservation law, the relevant experiments and theories can be roughly divided into two classes. Nonconserved systems can often be described by local equations of motion, such as the EdwardsWilkinson [1] or Kardar-Parisi-Zhang [2] equations. These models have a noise term with quenched or annealed correlations depending on the length scale of observation and the interface velocity [3]. On the other hand, processes involving the dynamics of phase boundaries coupled with conserved density fields are much less well understood. A good example of a problem in this class is imbibition, i.e., the propagation of liquid into a porous medium under the influence of capillary forces. Recent studies addressed [4-8] the propagation of an interface for spontaneous imbibition under the influence of capillary forces alone. In this case, the interface associated with the liquid-gas phase boundary typically moves according to Washburn law [9], where the average interface height $H(t) \propto t^{1 / 2}$. This idealized setup is hard to realize in experiments due to evaporation and gravity $[6,10-$ 13]. These complications qualitatively change the phenomenon, since both provide a maximal value ("pinning height"') for the distance between the average interface and the liquid reservoir. Evaporation moreover leads to a breakdown of the liquid conservation law.

It is thus important to develop an understanding of these external effects on the roughening process of the fluid front. The effect of a pinning mechanism on imbibition fronts was already addressed some time ago through the directed percolation depinning (DPD) model [10], including simultaneous work on experiments in which the pinning of an ink-water solution front in paper sheets was due to gravity. Later the model was extended to deal with the effect of evaporation [12] and, for a pinned interface, the experimental dependence of the width on the pinning height, $w \sim H_{p}^{\gamma}$ with $\gamma=0.49$ was reproduced. In spite of such successes, the dynamical processes of DPD do not include a conservation law, and as such cannot describe the early time behavior of spontaneous imbibition according to Washburn law. Other experiments $[6,14]$ presented a more complex picture of pinning, making the inclusion of physical pinning mechanisms in models of spontaneous imbibition [4-8] an interesting issue, this in order to explore both the pinned regime and it's approach.

Pinning of interfaces in nonconserved systems is well understood [15-17]. Close to pinning, these systems are characterized by a diverging length scale $\xi \sim\left(F-F_{c}\right)^{-\nu}$, where $F$ is the driving force of the interface, $F_{c}$ is the critical pinning force, and $\nu$ the correlation length exponent associated with $\xi$. Likewise, the velocity of the average interface follows $v \sim\left(F-F_{c}\right)^{\theta}$, where $\theta$ is the velocity exponent. The associated scaling exponent for surface roughness becomes $\chi=1.25$ or 0.633 in the isotropic and anisotropic cases, respectively. Some systems with a conservation law have also been studied. The forced slow propagation of a fluid in an Hele-Shaw cell gave results falling roughly within the quenched Edwards-Wilkinson universality class with a crossover to annealed noise at large flow rates [18]. Laplacian flow [19] and the depinning of a contact line [20,21] are also examples of systems in which the conservation law plays a role.

In this paper the pinning effects due to evaporation and gravity are studied by a generalization of the phase field model of spontaneous imbibition introduced in Refs. [5,7]. The mean field pinning limit and behavior of the average interface are described and an equation for the interface fluctuations is introduced. It is found that the inclusion of these external effects introduces additional length scales that control the spatial extent of roughening at pinning. The approach of the average interface to the pinning position is well described by a mean field theory and does not display any critical scaling of the type $v \sim\left(F-F_{c}\right)^{\theta}$, in contrast to theories of pinning for local models (see, e.g., Ref. [17]). The 
cases of external gravity and evaporation are then treated separately. When pinning is due to gravity alone there is a unique interface correlation length. For evaporation, a different correlation length controls the range of the fluctuations at pinning. In both cases, the correlation length can be obtained from the interfacial equation, as confirmed by numerical simulations. Furthermore, the interface is always found to be "superrough," with a global roughness exponent $\chi=1.25$. This is analogous to what is observed in the freely rising case, in contrast to the previously suggested DPD type of behavior $[10,12]$.

\section{PHASE FIELD APPROACH TO IMBIBITION}

A coarse-grained description of liquid flow in imbibition can be done in terms of a phase field $\phi(\mathbf{x}, \tau)$ taking characteristic values in the liquid and gas phase. Evaporation and gravity can be incorporated into a phase field model of imbibition developed previously by including nonconserving and convective terms, respectively [7]. The equation for the "order parameter" $\phi(\mathbf{x}, \tau)$ is then given by

$$
\frac{\partial \widetilde{\phi}(\mathbf{r}, \tau)}{\partial \tau}-\widetilde{G} \frac{\partial \widetilde{\phi}(\mathbf{r}, t)}{\partial y}=\nabla M(\widetilde{\phi}) \nabla \frac{\delta F}{\delta \widetilde{\phi}}-\frac{\tilde{\epsilon}}{2}\left[\phi_{e}+\widetilde{\phi}(\mathbf{r}, \tau)\right],
$$

with the free energy functional

$$
F=\frac{1}{2} \int d \mathbf{r}\left[r \widetilde{\phi}^{2}+\frac{u}{2} \widetilde{\phi}^{4}+\kappa(\boldsymbol{\nabla} \widetilde{\phi})^{2}-\tilde{\alpha}(\mathbf{r}) \widetilde{\phi}\right],
$$

where the equilibrium value $\phi_{e}=(r / u)^{1 / 2}$ represents the liquid $\left(+\phi_{e}\right)$ and gas $\left(-\phi_{e}\right)$ phases, and $\tilde{\alpha}$ sets the value of the local equilibrium chemical potential. Assuming a constant mobility $M$, these equations can be put in a dimensionless form by defining

$$
\begin{gathered}
\mathbf{x}=\mathbf{r} / \zeta, \quad \alpha=\left[\frac{u}{r^{3}}\right]^{1 / 2} \tilde{\alpha}, \\
t=\left[\frac{M r^{2}}{\kappa}\right] \tau, \quad g=\left[\frac{\kappa}{r^{3}}\right]^{1 / 2} \frac{\widetilde{G}}{M}, \\
\phi=\frac{\widetilde{\phi}}{\phi_{e}}, \quad \epsilon=\frac{\kappa}{M r^{2}} \tilde{\epsilon} .
\end{gathered}
$$

The ratio $\zeta=(\kappa / r)^{1 / 2}$ determines the width of the interfaces between the different phases. The corresponding phase field equation now has the form

$$
\partial_{t} \phi(\mathbf{x}, t)-g \partial_{y} \phi(\mathbf{x}, t)=\nabla^{2} \mu-\frac{1}{2} \epsilon(1+\phi(\mathbf{x}, t)),
$$

where the chemical potential $\mu=-\phi+\phi^{3}-\nabla^{2} \phi-\alpha(\mathbf{x})$. The model must still be supplemented with proper boundary conditions, chosen in the following way. At the bottom edge $(y=0)$, the presence of an infinite reservoir of "liquid" is reflected by the nonequilibrium value of $\mu(x, y=0)=$ const
$=0$. This value is arbitrary, since only differences in the chemical potential are relevant. Mass flow into the system from the top boundary is prevented through $\partial_{y} \mu\left(x, y=L_{y}\right)$ $=0$ and periodic boundary conditions in the $x$ direction are imposed.

The one-dimensional interface between the liquid and gas phases at equilibrium is represented by the kink solution $\phi_{0}(x, t)=\tanh (x / \sqrt{2})$. The quenched random field $\alpha(\mathbf{x})$ of mean $\bar{\alpha}$ and spatial correlations $\left\langle\alpha(\mathbf{x}) \alpha\left(\mathbf{x}^{\prime}\right)\right\rangle-\bar{\alpha}^{2}$ $=(\Delta \alpha)^{2} \delta\left(\mathbf{x}-\mathbf{x}^{\prime}\right)$ represents capillary forces on a coarsegrained scale. The evaporation term describes a loss of liquid $(\phi=+1)$ proportional to the area invaded, while the convective term (describing gravity) sets a limiting value for the gradient of the chemical potential.

\section{Mean field analysis}

Using projection techniques [22] a nonlocal interface equation can be obtained. Assuming a single-valued interface $h(x, t)$, the average position $H \equiv\langle h(x, t)\rangle$ is first obtained as

$$
\frac{d H(t)}{d t}=\frac{\bar{\alpha}}{2 H(t)}-g-\frac{1}{4} \epsilon H(t),
$$

which yields a pinning height $H_{p}(g, \epsilon)$ given by the zero of the right-hand side of Eq. (5) with limiting cases $H_{p}(g, \epsilon$ $=0)=\bar{\alpha} /(2 g)$ and $H_{p}(g=0, \epsilon)=\sqrt{2 \bar{\alpha} / \epsilon}$.

At early times $t \ll t^{*} \equiv \min \left(\bar{\alpha} g^{-2}, \epsilon^{-1}\right)$, the rise of the interface follows a Washburn behavior, $H(t)=(\bar{\alpha} t)^{1 / 2}$. For $t$ $>t^{*}$, the pinning height is approached exponentially slowly. In cases where gravity is absent, the average interface, of initial height $H(t=0)=0$, is described at all times by

$$
\left(\frac{H(t)}{H_{p}}\right)^{2}=1-e^{-\epsilon t / 2},
$$

while for $\epsilon=0$, the rise is described by the transcendental equation

$$
\frac{H(t)}{H_{p}}+\ln \left(1-\frac{H(t)}{H_{p}}\right)=-\frac{2}{\bar{\alpha}} g^{2} t .
$$

Although both gravity and evaporation pin the interface at a given height, it must be kept in mind that the physics behind these two effects is quite different [7]. Spontaneous imbibition, without external influence, is characterized by a Laplace equation for the chemical potential $\nabla^{2} \mu=0$ in the bulk (i.e., far away from the interface). When solved with boundary conditions $\mu(y=0)=0$ and $\mu(y=H)=-\bar{\alpha}$, this yields a gradient $\partial \mu \propto-1 / H$ in the liquid phase. The gravity term of Eq. (4) thus has the effect of stopping the interface when $\partial \mu \simeq-g$.

On the other hand, the nonconserving term introduced by evaporation is such that the chemical potential in the bulk must be a solution of the Poisson equation $\nabla^{2} \mu=\epsilon$ and at pinning height, the gradient in the chemical potential $\partial \mu$ 
$=0$. This simply represents the fact that the flux of liquid from the reservoir exactly balances the losses due to evaporation.

The time and length scales coming from gravity and evaporation can, however, be very different. Studies of capillary rise with light organic liquids (for which evaporation is presumably an extremely weak effect compared to ordinary water) imbibed in filter papers [23] have found a pinning height of the order of $1 \mathrm{~m}$, with time scales on the order of days. In contrast, evaporation effects can cause the pinning height to be of the order of $15-50 \mathrm{~cm}$, with correspondingly much faster time scales.

\section{EFFECTS OF DISORDER}

In the presence of quenched disorder, it can be expected that the actual pinning heights are slightly below the mean field predictions. Based on the picture developed for nonconserved fields, we can expect corrections $O\left(\Delta \alpha^{1 / 2}\right)$. For all simulations considered here, it is found that the approach of the mean interface towards pinning is well described by the mean-field result [Eqs. (6) and (7)], provided that the real pinning height is used, no signs of power law approach to pinning [21] are found. We also note that the present model predicts a pinning height due to evaporation $H_{p} \sim \epsilon^{-1 / 2}$, in opposition to the phenomenology of the DPD model [12].

A linearized interfacial equation can be obtained in terms of the Fourier components $h_{k}$ of the interface position $h(x, t)$. For nonzero Fourier modes, we obtain

$$
\begin{aligned}
& \left(\dot{h}_{k}+\frac{1}{2} \epsilon h_{k}\right)\left(1-e^{-2|k| H}\right)+|k|(\dot{H}+g) h_{k}\left(1+e^{-2|k| H}\right) \\
& \quad=\frac{1}{4}|k|\left(\{\eta\}_{k}-\sigma k^{2} h_{k}\right) .
\end{aligned}
$$

The quenched noise $\{\eta(t)\}_{k} \equiv \int_{x} e^{-i k x} \eta(x, h(x, t))$ where $\eta(x, h) \equiv \int d y \phi_{0}^{\prime}(y-h(x, t)) \alpha(x, y) \sim 2 \alpha(x, h)$ in the sharp interface limit. Immediately apparent is the presence of terms in odd power of wavevector $|k|$ arising from the conservation law.

Since the early time rise of the interface is consistent with Washburn behavior, we expect that the picture developed for spontaneous imbibition without external influence will apply at times $t \ll t^{*}$ [5]. In this case, we recall that the correlation length

$$
\xi_{\times}(t)=\frac{1}{2}\left(\frac{\sigma}{2 \dot{H}}\right)^{1 / 2}=\frac{1}{2}\left(\frac{\sigma H(t)}{\bar{\alpha}}\right)^{1 / 2}
$$

controls the spatial extent of correlated interfacial fluctuations. In particular, the interfacial width $W^{2}(t)$ $=\left\langle\overline{(h(x, t)-H(t))^{2}}\right\rangle$, where the brackets denote an average over different realizations of $\alpha$ and the overbar a spatial average over the system, has an early time behavior $W(t)$ $\sim \xi_{\times}^{\chi} \sim t^{\chi / 4}$ with the roughness exponent $\chi=1.25$. The structure factor $S(k, t)=\left\langle\overline{h_{k}(t) h_{-k}(t)}\right\rangle$ can then be shown to have the scaling form

$$
S(k, t)=\left(\xi_{\times}(t)\right)^{1+2 \chi_{S}}\left(k \xi_{\times}(t)\right),
$$

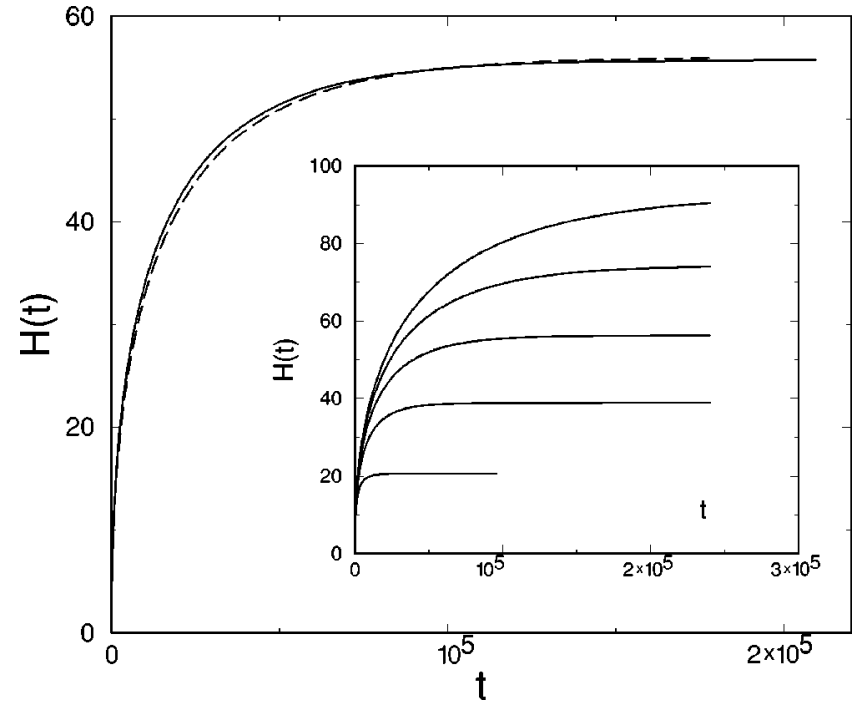

FIG. 1. Behavior of the average interface height $H(t)$ in the gravity dominated case with $\bar{\alpha}=0.2$. The main figure compares the heights obtained from numerical simulations for $g=0.00167$ (dashed line; $H_{p} \approx 60$ ) to the the mean-field results of Eq. (7) (solid line, using an effective pinning height $H_{p}=56$ ). In the inset, we show $H(t)$ for values of $g$ corresponding to theoretical pinning heights $H_{p}=20,40,60,80$, and 100 . For the last value, the interface has not yet reached the pinning limit. All quantities are in the dimensionless units of Eq. (4).

with the scaling function $s(x \gg 1) \sim x^{-1-2 \chi}$, and becomes constant for $x \ll 1$. The correlation function $G_{2}(r, t)$ $=\left\langle\overline{|h(x+r, t)-h(x, t)|^{2}}\right\rangle^{1 / 2}$ consequently has a scaling form $G_{2}(r, t)=\xi_{\times}^{\chi} g\left(r / \xi_{\times}\right)$, where $g(x \ll 1) \sim u$ (anomalous scaling [24]), with $g(x \gg 1)$ tending to a constant.

In contrast to ordinary models of kinetic roughening, this new length scale is not a genuine (time dependent) correlation length that would diverge in power law fashion with increasing time. The function of $\xi_{\times}$is to restrict the spatial extent of fluctuations [12], and therefore the interface cannot be considered self-affine on all length scales.

In presence of pinning effects, this behavior becomes modified at later times. The behavior of the interface fluctuations as well as the range of the scaling close to pinning is quite different, depending on whether gravity or evaporation is the dominant external influence. Below, these two cases are treated separately.

\section{A. Gravity dominated case}

Setting first $\epsilon=0$ allows us to consider the dynamical rise in presence of gravity alone. As seen from Fig. 1, the behavior of the average interface height is well described by the transcendental equation [Eq. (7)], with the only proviso that an effective pinning height is used instead of the mean field value.

In the absence of evaporation, the linearized equation of the fluctuations [Eq. (8)], immediately shows the existence of a length scale $\xi_{g}(t)$, restricting the range of the spatial fluctuations and evolving in time as 


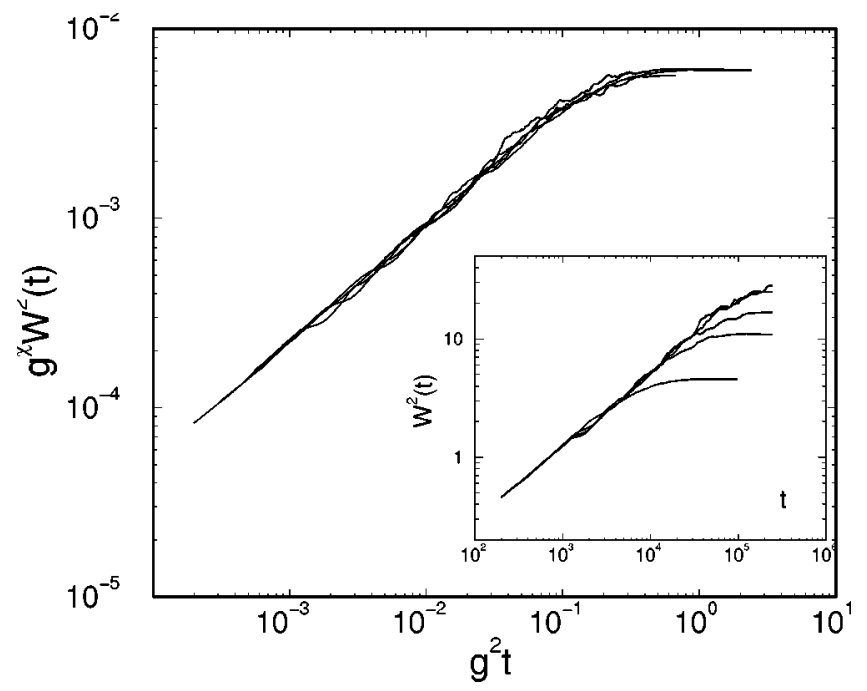

FIG. 2. Behavior of the interface width as a function of time for imbibition in the presence of gravity. The inset shows the rms width for systems with values of $g$ corresponding to pinning heights $H_{p}$ $=20,40,60,80$, and 100 (bottom to top). The main figure shows the result of the scaling assumption of Eq. (13), with the value $\chi$ $=1.25$. All quantities are in the dimensionless units of Eq. (4).

$$
\xi_{g}(t)=\frac{1}{2}\left(\frac{\sigma}{2(\dot{H}+g)}\right)^{1 / 2}=\frac{1}{2}\left(\frac{\sigma H(t)}{\bar{\alpha}}\right)^{1 / 2},
$$

where $H(t)$ is given by the solution of Eq. (7) [minor corrections of order $O\left(\xi_{g} / H\right)$ are also expected]. The length scale $\xi_{g}(t)$ is thus analogous to the length scale $\xi_{\times}(t)$ in spontaneous imbibition without external influences, although it is not a simply power law in time any more. At pinning, this length scale becomes

$$
\xi_{g}\left(H_{p}\right)=\frac{1}{2}\left(\frac{\sigma}{2 g}\right)^{1 / 2}=\xi_{\times}\left(H_{p}\right) .
$$

As in spontaneous imbibition without gravity, dynamical scaling relations can be established by assuming a single correlation length $\xi_{g}(t)$. Equation (11) can first be rewritten as $\xi_{g}(t)=0.25 g^{-1 / 2}\left(\sigma H / H_{p}\right)^{1 / 2}$. Since, from Eq. (7), the quantity $H / H_{p}$ is a function of $g^{2} t$ only, the temporal behavior of the width of the interface $W(t)$ can be scaled as

$$
W(t)=g^{-\chi / 2} w\left(g^{2} t\right),
$$

where $w(x) \sim x^{\chi / 4}$ for $x \ll 1$ and tends to a constant for large arguments, as represented in Fig. 2. The global roughness exponent $\chi=1.25$ has the same value as for spontaneous imbibition without external influences.

The value of the roughness exponent as well as the role of the correlation length $\xi_{g}$ at pinning is confirmed from data for the structure factor. Figure 3 shows the structure factor for systems with different pinning heights (arising from different values of the constant $g$ ). The structure factor decays as $k^{-3.5}$ at large values of the wave vector, consistent with the value $\chi=1.25$, and the curves can be collapsed on the common scaling form by setting $\xi_{g}\left(H_{p}\right) \sim g^{-1 / 2} \sim H_{p}^{1 / 2}$ :

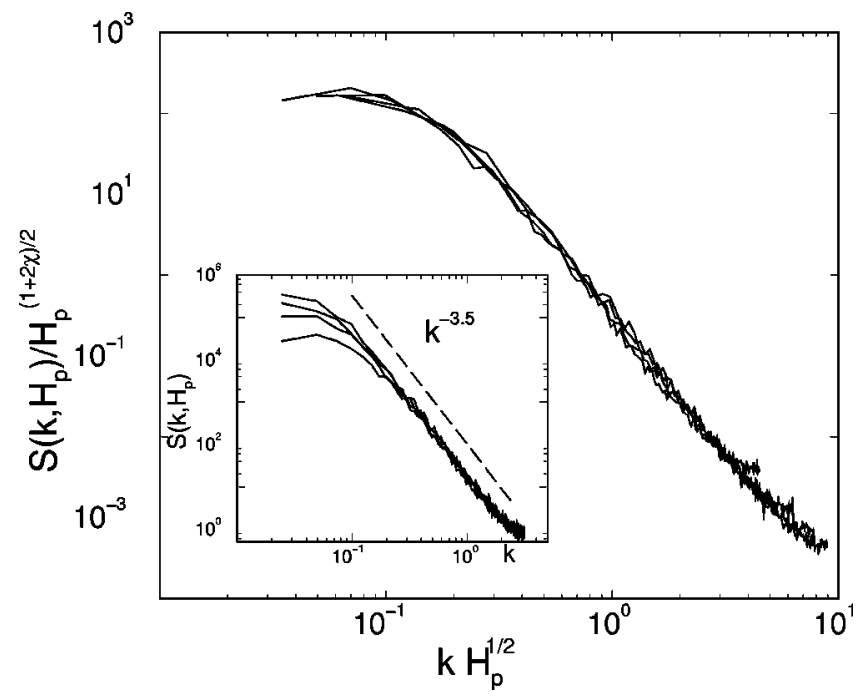

FIG. 3. A log-log plot of structure factors of the pinned interface in the presence of gravity. The inset shows the value of $S\left(k, H_{p}\right)$ for pinning heights $H_{p}=20,40,60$, and 80 . The main figure shows the collapse of the data under the assumption of a correlation length $\xi_{g}\left(H_{p}\right) \sim H_{p}^{1 / 2}$. The dashed line indicates a roughness exponent of $\chi=1.25$. All quantities are in the dimensionless units of Eq. (4).

$$
S\left(k, H_{p}\right)=\left(\xi_{g}\left(H_{p}\right)\right)^{1+2 \chi_{S}}\left(k \xi_{g}\left(H_{p}\right)\right) .
$$

The scaling function $s(k \xi)$ is the same as in Eq. (10), and the notation $\xi_{g}\left(H_{p}\right)$ indicates that the structure factor is considered at "pinning." Similar scaling relations also apply to the two-point correlation function $G_{2}(r, t)$, which now also shows anomalous scaling [24].

As for pure spontaneous imbibition the concept of a dynamical exponent $z$ describing the propagation of fluctuations along the interface does not apply to imbibition with gravity. The fluctuations always catch up to the available zone of correlated roughness. An effective temporal exponent $\beta$ such that $W(t) \sim t^{\beta}$ is apparent only for times $t$ $\ll \bar{\alpha} g^{-2}$, in which case it takes the value $\beta \sim 0.31[5,7]$.

\section{B. Evaporation dominated case}

In the presence of evaporation (and absence of gravity) the interface equation [Eq. (8)] has a more complex structure. As in the case with gravity the average interface dynamics is well described by the mean-field equation as shown in Fig. 4, and again leads to a pinning height that depends weakly on the disorder strength. However, due to the term in $\epsilon\left(1-e^{-2|k| H}\right)$, a single correlation length $\xi$ $=\xi(t, H(t))$ cannot be unambiguously identified for the kinetic roughening.

The situation becomes clear only in the pinned interface limit, where a simple correlation length can be defined as

$$
\sigma \xi_{e}^{-3}=2 \epsilon\left(1-e^{-2 H_{p} / \xi_{e}}\right) .
$$

In the limit $\xi_{e} \ll H_{p}, \xi_{e} \sim(\sigma / \epsilon)^{1 / 3}$ while in the opposite limit $\xi_{e} \gg H_{p}, \quad \xi_{e} \sim\left(\sigma^{2} /(\bar{\alpha} \epsilon)\right)^{1 / 4}$. The first case corresponds to weak evaporation, defined by $\epsilon \ll \bar{\alpha}^{3} / \sigma^{2}$, while the second 


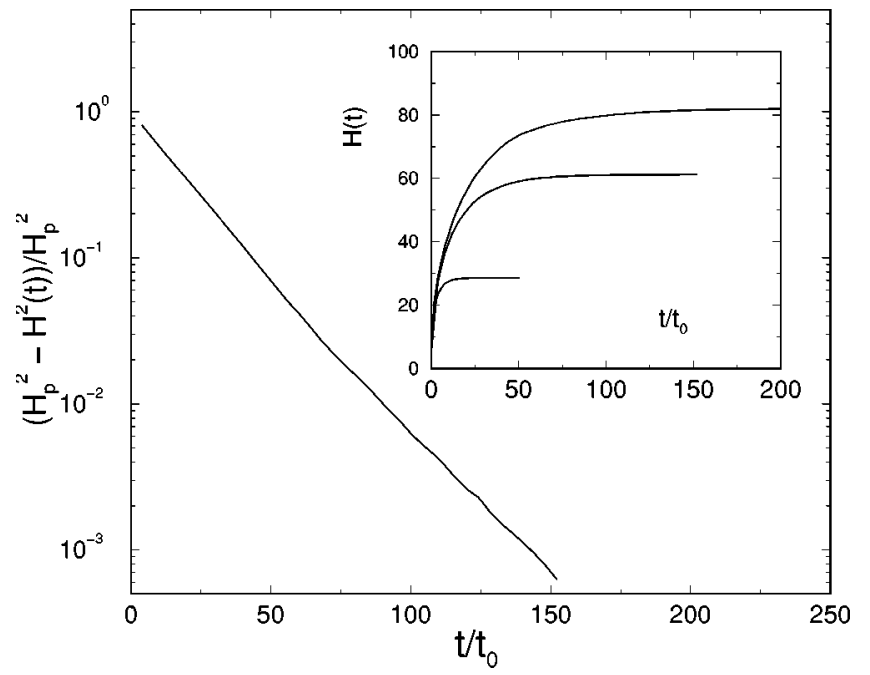

FIG. 4. Behavior of $H(t)$ in the case where evaporation is the dominant effect. The parameters chosen are $\epsilon=5 \times 10^{-3}, 10^{-4}$, and $5 \times 10^{-5}$, with $\bar{\alpha}=0.2$. The main figure shows the exponential approach to pinning as predicted by Eq. (6) for $\epsilon=10^{-4}$. The curve has a slope of $4.7 \times 10^{-5} \approx \epsilon / 2$. The scale factor $t_{0}=1000$ in the dimensionless units of Eq. (4). All quantities are in the dimensionless units of Eq. (4).

case is that of strong evaporation, $\epsilon \gg \bar{\alpha}^{3} / \sigma^{2}$. This argument indicates the range of correlated roughness but does not specify the value of the roughness exponents. However, this can be established from the decay of the structure factor, again with the result $\chi=1.25$.

The prediction for the pinned correlation length can be checked by considering the scaling behavior of the pinned structure factors $S\left(k, H_{p}\right)$, as shown in Fig. 5. The data with the highest evaporation rate can all be collapsed assuming a correlation length $\xi_{e} \sim(\bar{\alpha} \epsilon)^{-1 / 4}$, while for lower evaporation $\xi_{e} \sim \epsilon^{-1 / 3}$. The crossover occurs roughly for parameters $\bar{\alpha}$ $=0.2$ and $\epsilon=10^{-4}$. The complete set of data can be reduced to a common scaling form by solving Eq. (15) numerically (using a value of $\sigma=2 \sqrt{2} / 3$ ). As a result the structure factor at pinning has a scaling form similar to Eq. (10), although care must be taken in identifying the correlation length.

Even though a clear dynamical correlation length cannot be unambiguously defined [25], the early time limit $t \ll \epsilon^{-1}$ can, however, be studied in order to extract all the scaling behavior already established in the case of imbibition without any external effect. Figure 6 shows the two-point correlation function of an interface with parameters $\bar{\alpha}=0.3$ and $\epsilon=5 \times 10^{-5}$. The data for times $t \ll \epsilon^{-1}=20000$ [in dimensionless units of Eq. (4)] can be collapsed on a single curve assuming $\xi_{\times} \sim t^{1 / 4}$. After this time, it is likely that the dynamical fluctuations of the interface are controlled by a genuine dynamical correlation length of the form $\xi_{t} \sim t^{1 / z}$, until a time where $\xi_{t} \sim \xi_{\epsilon}$. A value of $z$ could not be obtained from the data, since a precise determination of the dynamical exponent would require pinning heights much higher than can be achieved numerically.

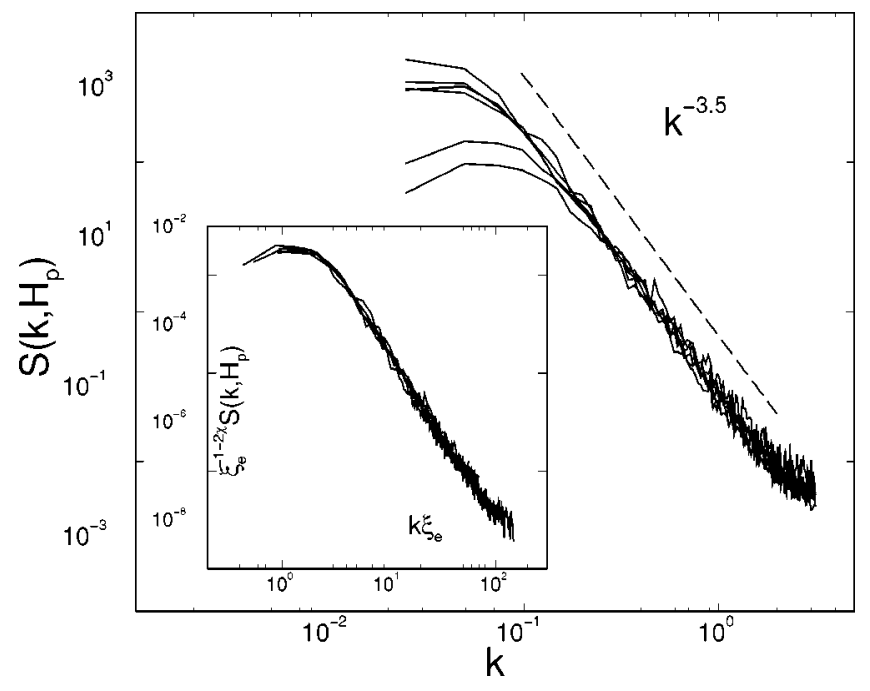

FIG. 5. A log-log plot of structure factors of the pinned interface in the presence of evaporation. The main figure shows the value of $S\left(k, H_{p}\right)$ for pinning heights (from bottom to top): $H_{p} \simeq 20$ ( $\bar{\alpha}$ $\left.=0.2, \epsilon=10^{-} 3\right) ; \quad H_{p} \simeq 28\left(\bar{\alpha}=0.2, \epsilon=5 \times 10^{-} 4\right) ; \quad H_{p} \simeq 45(\bar{\alpha}$ $\left.=0.1, \epsilon=10^{-} 4\right) ; H_{p} \simeq 63\left(\bar{\alpha}=0.2, \epsilon=10^{-} 4\right) ; H_{p} \simeq 77 \quad(\bar{\alpha}=0.3, \epsilon$ $\left.=10^{-} 4\right) ; H_{p} \simeq 109\left(\bar{\alpha}=0.3, \epsilon=5 \times 10^{-} 5\right)$. The structure factors for $\epsilon \sim 10^{-4}$ all collapse on the same curve, independent of the value of $\bar{\alpha}$. A complete collapse of the data can be accomplished by solving Eq. (15) numerically, as shown in the inset. The dashed line indicates a roughness exponent of $\chi=1.25$. All quantities are in the dimensionless units of Eq. (4).

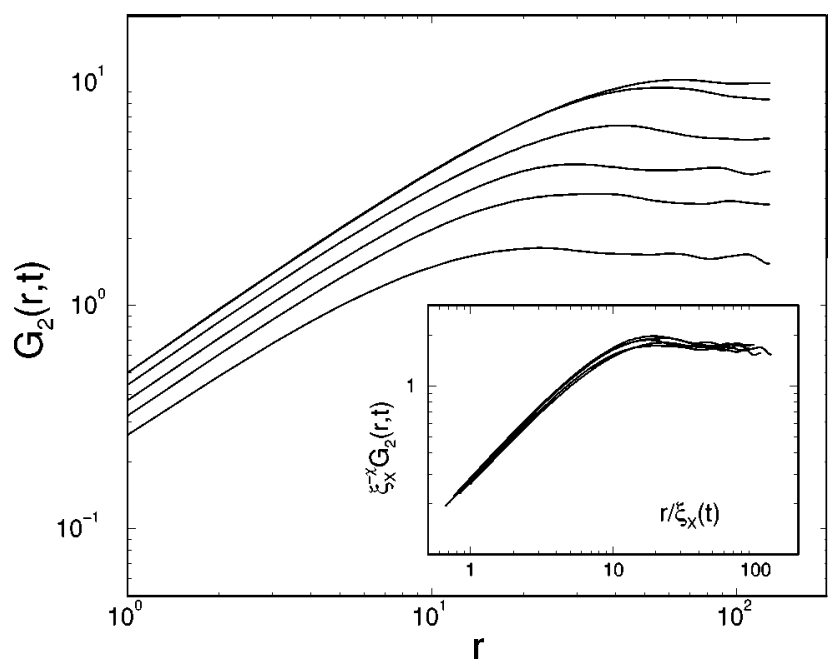

FIG. 6. Temporal behavior of $G_{2}(r, t)$ for imbibition in the presence of evaporation. The main figure is for $\bar{\alpha}=0.3$ and $\epsilon=5$ $\times 10^{-5}$ at times (from bottom to top) $t=2 \times 10^{3}, 10^{4}, 2 \times 10^{4}, 4$ $\times 10^{4}, 10^{5}$, and $3 \times 10^{5}$, after which $G_{2}(r, t)$ no longer changes. In the inset, we show the early time scaling of the correlation function under the assumption $\xi_{\times}(t) \sim t^{1 / 4}$. The collapse of the data is shown for times $t=2 \times 10^{3}$ to $10^{4}$ at intervals of $2 \times 10^{3}$. The value of $\chi$ $=1.25$. All quantities are in the dimensionless units of Eq. (4). 


\section{SUMMARY AND CONCLUSIONS}

The experimental study of imbibition is very delicate, due to intrinsic difficulties such as the very slow time scale of the interface motion and the finite region available to correlated fluctuations. It is thus important to understand and control external effects such as gravity and evaporation. The theory presented above makes definite predictions about the pinning height of the interface, and sets an upper time limit on the region over which the theories of spontaneous imbibition without external influence should be valid. Beyond this regime the interface properties are determined by the physical mechanism (evaporation or gravity) that leads to the eventual pinning. We note that in spite of the different temporal behavior of the interface height as compared to the spontaneous imbibition case [5,7], the correlated fluctuations follow a similar roughening picture here. That is, the global roughness exponent $\chi$ also retains its "superrough" value of about 1.25 in the case where either gravity or evaporation dominates.

Although a precise quantification and control of the evaporation rate can be extremely difficult, the dynamical progression of average interface height can be used to extract both the pinning height $H_{p}$ and the effective evaporation rate $\epsilon$. Since the time scales associated with evaporation are rela- tively fast, it becomes interesting to test the predictions of the model. For this one can use the value of the correlation length at pinning, related to the saturated width $w_{s a t} \sim H_{p}^{\gamma}$, with $\gamma=2 \chi / 3=0.83$ for weak evaporation and $\gamma=\chi / 2$ $=0.75$ for strong evaporation. Both these expressions differ from Amaral et al.'s experiment with ink [12]. However, a precise measurement requires a very good control of $\epsilon$, and that the interface roughness develops on appreciable length scale [6].

If evaporation is likely to be a major effect for imbibition of low-surface tension liquids such as water, its effect can be greatly reduced by using low-molecular weight organic liquids. Gravity, whose effective value can be changed by performing the imbibition experiment at various inclinations, is then the dominant external effect. The prediction of Eq. (11) that the interfacial fluctuations can be described by a single correlation length $\xi_{g}(t)$, although with a nontrivial time dependence, can then also be checked experimentally.

\section{ACKNOWLEDGMENTS}

This work was supported by Research Corporation Grant No. CC4787 (K.R.E.) and NSF-DMR Grant No. 0076054 (K.R.E.), and the Academy of Finland though its Center of Excellence and MADAME programs.
[1] S. F. Edwards and D. R. Wilkinson, Proc. R. Soc. London, Ser. A 281, 17 (1982).

[2] M. Kardar, G. Parisi, and Y.-C. Zhang, Phys. Rev. Lett. 56, 889 (1986).

[3] L. A. N. Amaral, A.-L. Barabási, H. A. Makse, and H. E. Stanley, Phys. Rev. E 52, 4087 (1995); S. Roux and A. Hansen, J. Phys. I 4, 515 (1994); M. Jost and K. D. Usadel, Phys. Rev. B 54, 9314 (1996).

[4] V. Ganesan and H. Brenner, Phys. Rev. Lett. 81, 578 (1998).

[5] M. Dubé, M. Rost, K. R. Elder, M. Alava, S. Majaniemi, and T. Ala-Nissila, Phys. Rev. Lett. 83, 1628 (1999).

[6] M. Dubé, M. Rost, and M. Alava, Eur. Phys. J. B 15, 691 (2000).

[7] M. Dubé, M. Rost, K. R. Elder, M. Alava, S. Majaniemi, and T. Ala-Nissila, Eur. Phys. J. B 15, 701 (2000).

[8] C.-H. Lam and V. K. Horváth, Phys. Rev. Lett. 85, 1238 (2000).

[9] E. W. Washburn, Phys. Rev. 17, 273 (1921); E. K. Rideal, Philos. Mag. 44, 1152 (1992).

[10] S. V. Buldyrev, A.-L. Barabási, F. Caserta, S. Havlin, H. E. Stanley, and T. Vicsek, Phys. Rev. A 45, R8313 (1992). Also see H. Leschhorn and L. H. Tang, Phys. Rev. E 49, 1238 (1994); H. Leschhorn, ibid. 54, 1313 (1996).

[11] F. Family, K. C. B. Chan, and J. G. Amar, in Surface Disordering: Growth, Roughening and Phase Transitions, edited by R. Jullien, J. Kertész, P. Meakin, and D. E. Wolf (Nova Science, Commack, NY, 1992).

[12] L. A. N. Amaral, A.-L. Barabási, S. V. Buldyrev, S. Havlin, and H. E. Stanley, Phys. Rev. Lett. 72, 641 (1994).

[13] V. K. Horváth and H. E. Stanley, Phys. Rev. E 52, 5166 (1995).

[14] P. B. S. Kumar and D. Jana, Physica A 224, 199 (1996).

[15] O. Narayan and D. S. Fisher, Phys. Rev. B 48, 7030 (1993).

[16] H. Leschhorn, Physica A 195, 324 (1993).

[17] H. Leschhorn, T. Nattermann, S. Stepanow, and L.-H. Tang, Ann. Phys. (Leipzig) 6, 1 (1997).

[18] S. He, G. L. M. K. S. Kahanda, and P.-z. Wong, Phys. Rev. Lett. 69, 3731 (1992); R. Albert, A.-L. Barabási, N. Carle, and A. Dougherty, ibid. 81, 2926 (1998).

[19] J. Krug and P. M. Meakin, Phys. Rev. Lett. 66, 703 (1991).

[20] J. F. Joanny and P. G. de Gennes, J. Chem. Phys. 81, 552 (1984); E. Rolley, C. Guthmann, T. Gombrowicz, and V. Repain, Phys. Rev. Lett. 80, 2865 (1998).

[21] T. Delker, D. B. Pengra, and P.-z. Wong, Phys. Rev. Lett. 76, 2902 (1996); E. Schaffer and P.-z. Wong, ibid. 80, 3069 (1998); Phys. Rev. E 61, 5257 (2000).

[22] K. Kawasaki and T. Ohta, Prog. Theor. Phys. 68, 129 (1982).

[23] T. Gillespie, J. Colloid Sci. 13, 32 (1958); 14, 123 (1959).

[24] J. M. López, M. A. Rodríguez, and R. Cuerno, Phys. Rev. E 56, 3993 (1997).

[25] Even in the case of strong evaporation, the approximation $|k| H \ll 1 \quad \forall|k|$, which results in a correlation length of the form $\xi_{\times}^{2} \sim \sigma /(\epsilon+2 H(t))[H(t)$ is the solution to the meanfield equation (6)], does not give an adequate description of the dynamical scaling behavior at all times. 